\title{
IDEALS OF SQUARE SUMMABLE POWER SERIES IN SEVERAL VARIABLES
}

\author{
JAMES RADLOW
}

\begin{abstract}
Let $\mathscr{C}(z)$ be the Hilbert space of formal power series in $z_{1}, \cdots, z_{r}(r \geqq 1)$. An ideal of $\mathscr{C}(z)$ is a vector subspace $\mathscr{M}$ of $\mathscr{C}(z)$ which contains $z_{1} f(z), \cdots, z_{r} f(z)$ whenever it contains $f(z)$. If $B(z)$ is a formal power series such that $B(z) f(z)$ belongs to $\mathscr{C}(z)$ and $\|B(z) f(z)\|=\|f(z)\|$, then the set $\mathscr{M}(B)$ of all products $B(z) f(z)$ is a closed ideal of $\mathscr{C}(z)$. In the case $r=1$, Beurling showed that every closed ideal is of this form for some such $B(z)$. Here we give conditions under which a closed ideal is of the form $\mathscr{M}(B)$ for $r \geqq 2$.
\end{abstract}

By a formal power series in $r \geqq 1$ variables we mean a formal sum $f(z)=$ $\sum a_{n} z^{n}$, where $a_{n}=a_{n_{1} \ldots n_{r}}$ is a complex number depending on the multiindex $n=n_{1} \cdots n_{r}$ and $z^{n}=z_{1}^{n}{ }_{1} \cdots z_{r}^{n_{r}}\left(n_{1}, \cdots, n_{r}=0,1,2, \cdots\right)$. Questions of convergence do not arise because we treat $z_{1}, \cdots, z_{r}$ as indeterminates. Let $\mathscr{C}(z)$ be the Hilbert space of formal power series $f(z)=\sum a_{n} z^{n}$ such that

$$
\|f(z)\|^{2}=\sum\left|a_{n}\right|^{2}<\infty .
$$

An ideal of $\mathscr{C}(z)$ is a vector subspace $\mathscr{M}$ of $\mathscr{C}(z)$ which contains $z_{1} f(z)$, $\cdots, z_{r} f(z)$ whenever it contains $f(z)$. Except for the case $r=1$, the closed ideals of $\mathscr{C}(z)$ are not known. For an example of a closed ideal, let $B(z)$ be a formal power series such that the formal product $B(z) f(z)$ belongs to $\mathscr{C}(z)$ and $\|B(z) f(z)\|=\|f(z)\|$ for every $f(z)$ in $\mathscr{C}(z)$. Then the set $\mathscr{M}(B)$ of all products $B(z) f(z)$, where $f(z)$ is in $\mathscr{C}(z)$, is a closed ideal of $\mathscr{C}(z)$. In the case $r=1$, it is a well-known fact that every closed ideal is of this form for some such series $B(z)$. The theorem was given by Beurling [1]. We are following the notation of Rovnyak ([4]; or see deBranges and Rovnyak [2, p. 12]) whose elementary proof of Beurling's theorem is here extended in a straightforward manner. The resulting theorem gives conditions under which a closed ideal is of the form $\mathscr{M}(B)$ for $r \geqq 2$.

LeMma 1. Let $B(z)$ be a formal power series. A necessary and sufficient condition that $B(z) f(z)$ belong to $\mathscr{C}(z)$ and $\|B(z) f(z)\|=\|f(z)\|$ for every $f(z)$ in $\mathscr{C}(z)$ is that $\left(z^{n} B(z)\right)$ be an orthonormal set in $\mathscr{C}(z)$. In this case the

Received by the editors May 29, 1967 and, in revised form, April 14, 1972 and July 31, 1972.

AMS (MOS) subject classifications (1970). Primary 47A15, 47A45; Secondary 32A05.

Key words and phrases. Hilbert space of formal power series, ideal, formal product, Beurling's theorem, projection, commutativity relations.

(C) American Mathematical Society 1973 
expansion $B(z) f(z)=\sum a_{n} z^{n} B(z)$, where $f(z)=\sum a_{n} z^{n}$, is valid in the metric of $\mathscr{C}(z)$.

LEMMA 2. Let $f(z)$ and $g(z)$ be formal power series such that $\left(z^{n} f(z)\right)$ and $\left(z^{n} g(z)\right)$ are orthonormal sets in $\mathscr{C}(z)$. If $\left\langle z^{m} f(z), z^{n} g(z)\right\rangle=0$ whenever $m \neq n$, then $f(z)$ and $g(z)$ are linearly dependent.

THEOREM. If $\mathscr{M}$ is a closed ideal of $\mathscr{C}(z)$ containing a nonzero element, let $P_{k}$ denote the projection of $\mathscr{M}$ into the set of series of the form $z_{k} f(z)$ with $f(z)$ in $\mathscr{M}(k=1, \cdots, r)$. A necessary and sufficient condition that $\mathscr{M}=$ $\mathscr{M}(B)$ for some series $B(z)$ such that $\left(z^{n} B(z)\right)$ is an orthonormal set in $\mathscr{C}(z)$ is that for every $f(z)$ in $\mathscr{M}$ the commutativity relations $P_{i} z_{j} f(z)=z_{j} P_{i} f(z)$ (with $i, j=1, \cdots, r$ and $i \neq j$ ) be valid. When $B(z)$ exists, it is unique to within a multiplicative constant of absolute value one.

Proof of Lemma 1. As in [4], it is convenient to introduce the notion of formal convergence. A sequence $\left(f_{j}(z)\right)$ of formal power series in $\mathscr{C}(z)$ is said to converge formally if for each multi-index $j=j_{1} \cdots j_{r}\left(j_{k}=0,1\right.$, $2, \cdots ; k=1, \cdots, r)$ the corresponding sequence of $j$ th coefficients converges. If a sequence converges in the metric of $\mathscr{C}(z)$, then it converges formally to the same limit.

Let $B(z)$ meet the conditions of the lemma. It is then easy to see that the series $\sum a_{n} z^{n} B(z)$ converges in the metric of $\mathscr{C}(z)$, its formal sum being $B(z) f(z)$. It follows that the equation $B(z) f(z)=\sum a_{n} z^{n} B(z)$ is valid in $\mathscr{C}(z)$, i.e. that $B(z) f(z)$ belongs to $\mathscr{C}(z)$ as claimed. The sufficiency proof is complete upon our noting that

$$
\begin{aligned}
\|B(z) f(z)\|^{2} & =\left\langle\sum a_{n} z^{n} B(z), \sum a_{m} z^{m} B(z)\right\rangle \\
& =\sum\left\langle a_{n} z^{n} B(z), a_{n} z^{n} B(z)\right\rangle=\sum\left|a_{n}\right|^{2}=\|f\|^{2} .
\end{aligned}
$$

To prove necessity, observe that the monomials $\left(z^{n}\right)$ form an orthonormal set in $\mathscr{C}(z)$. Since an isometry preserves inner products, $\left\langle z^{m} B(z), z^{n} B(z)\right\rangle=$ $\delta_{m n}$.

Proof of Lemma 2. The conditions imposed on $B(z)$ in Lemma 1 are met by $f(z)$ and $g(z)$. Thus, $f(z) g(z)$ is in $\mathscr{C}(z)$, while $\|f g\|=\|f\| \cdot\|g\|=1$. If $a_{n}, b_{n}$ are the coefficients of $f, g$ respectively, then $f(z) g(z)=\sum b_{n} z^{n} f(z)$, $g(z) f(z)=\sum a_{n} z^{n} g(z)$ are valid equations in $\mathscr{C}(z)$. Making use of the conditions on $f(z)$ and $g(z)$, together with the fact that multiplication by $z^{n}$ is isometric, we find:

$$
\begin{aligned}
\|f(z) g(z)\|^{2} & =\langle f(z) g(z), g(z) f(z)\rangle=\left\langle\sum b_{m} z^{m} f(z), \sum a_{n} z^{n} g(z)\right\rangle \\
& =\sum\left\langle b_{n} f(z), a_{n} g(z)\right\rangle=\langle f(z), g(z)\rangle \cdot \sum b_{n} \bar{a}_{n} \\
& =\langle f(z), g(z)\rangle \cdot\langle g(z), f(z)\rangle .
\end{aligned}
$$


The result is that

$$
|\langle f(z), g(z)\rangle|^{2}=1=\|f(z)\|^{2} \cdot\|g(z)\|^{2},
$$

the Schwarz inequality therewith reducing to an equality, and this implies that $f(z)$ and $g(z)$ are linearly dependent.

ProOF OF TheOREM. Let $\mathscr{M}=\mathscr{M}(B)$, where $\left(z^{n} B(z)\right)$ is an orthonormal set in $\mathscr{C}(z)$, and let $f(z)$ belong to $\mathscr{M}(B)$. Then necessity is established (as the reader may easily verify) by computing both $z_{j} P_{i} f(z)$ and $P_{i} z_{j} f(z)$ and observing that they are equal when $i \neq j$.

Now denote by $\mathscr{N}_{k}$ the set of series $z_{k} f(z)$ for $f(z)$ in $\mathscr{M}(k=1, \cdots, r)$. Since multiplication by $z_{k}$ is isometric, while $\mathscr{M}$ is an ideal, each $\mathscr{N}_{k}$ is a subspace contained in $\mathscr{M}$. The closed span $\bigvee_{k} \mathscr{N}_{k}=\mathscr{N}$ is again a subspace, and $\mathscr{N} \subseteq \mathscr{M}$. To prove that $\mathscr{N} \neq \mathscr{M}$, we first show that $P_{i} P_{j}=P_{j} P_{i}$ for all $i, j=1, \cdots, r$. The assertion is trivial if $i=j$. If $i \neq j$, let $f(z)=z_{i} g(z)$ for some $g(z)$ in $\mathscr{M}$. Then

$$
\begin{aligned}
& P_{i} P_{j} f(z)=P_{i} P_{j} z_{i} g(z)=P_{i} z_{i} P_{j} g(z)=z_{i} P_{j} g(z), \\
& P_{j} P_{i} f(z)=P_{j} P_{i} z_{i} g(z)=P_{j} z_{i} g(z)=z_{i} P_{j} g(z),
\end{aligned}
$$

and similarly for $f(z)=z_{j} g(z)$. Thus $P_{i} P_{j}$ and $P_{j} P_{i}$ coincide on $\mathscr{N}_{i} \vee \mathscr{N}_{j}$. Since $P_{i}$ and $P_{j}$ vanish on the complement of $\mathscr{N}_{i} \vee \mathscr{N}_{j}$ in $\mathscr{M}$, we have $P_{i} P_{j}=P_{j} P_{i}$ for $i, j=1, \cdots, r$. If $r=2$, this implies [3, vol. II, p. 55] that the projection operator $P=P_{1} \vee P_{2}$ of $\mathscr{M}$ onto $\mathscr{N}=\mathscr{N}_{1} \vee \mathscr{N}_{2}$ is given by

$$
P=1-\left(1-P_{1}\right)\left(1-P_{2}\right)=P_{1}+P_{2}-P_{1} P_{2} \text {. }
$$

For $r>2$, it is easy to see that

$$
P=1-\left(1-P_{1}\right) \cdots\left(1-P_{r}\right) .
$$

To save writing, we take $r=2$ for the moment.

If $g(z)$ is in $\mathscr{N}$, we then have

$$
g(z)=P g(z)=P_{1} g(z)+P_{2} g(z)-P_{1} P_{2} g(z) .
$$

From the definition of the operator $P_{k}, P_{1} g(z)=z_{1} g_{1}(z), P_{2} g(z)=z_{2} g_{2}(z)$ for some series $g_{1}(z), g_{2}(z)$ in $\mathscr{M}$. The hypothesis $P_{1} z_{2} g(z)=z_{2} P_{1} g(z)$ gives

$$
P_{1} P_{2} g(z)=P_{1} z_{2} g_{2}(z)=z_{2} P_{1} g_{2}(z)=z_{1} z_{2} g_{12}(z)
$$

for some $g_{12}(z)$ in $\mathscr{M}$. Every $g(z)$ in $\mathscr{N}$ is therefore of the form

$$
g(z)=z_{1} g_{1}(z)+z_{2} g_{2}(z)-z_{1} z_{2} g_{12}(z)
$$

where $g_{1}(z), g_{2}(z)$ and $g_{12}(z)$ are in $\mathscr{M}$.

To complete the proof that $\mathscr{M} \neq \mathscr{N}$, we suppose the contrary: that any $g(z)$ in $\mathscr{M}$ is in $\mathscr{N}$. The representation (3) therefore holds not only for 
$g(z)$ but also for $g_{1}(z), g_{2}(z)$ and $g_{12}(z)$. By repeating the argument $n$ times we see that

$$
g(z)=\sum p_{\alpha}(z) g_{\alpha}(z)
$$

where $p_{\alpha}(z)$ is a monomial of degree $\geqq n$ and $g_{\alpha}(z) \in \mathscr{M}$ for all $\alpha$. Since $n$ is arbitrary, every $g(z)$ in $\mathscr{M}$ vanishes identically if $\mathscr{M}=\mathscr{N}$. We conclude that $\mathscr{M} \neq \mathscr{N}$. The reasoning holds for $r=2$. For $r>2$, we merely take $\left(1^{\prime}\right)$ instead of (1) as our starting point.

Let $\mathscr{B}$ denote the orthogonal complement of $\mathscr{N}$ in $\mathscr{M}$. Notice that $\mathscr{B}=\bigcap_{k} \mathscr{B}_{k}$, where $\mathscr{B}_{k}=\mathscr{N}_{k} \frac{1}{k} \cap \mathscr{M}$. Having shown that the subspace $\mathscr{N} \neq \mathscr{M}$, we may assert that $\mathscr{B}$ contains a nonzero element. Denote by $B(z)$ an element of $\mathscr{B}$ having unit norm. Since $B(z)$ belongs to each of the subspaces $\mathscr{B}_{k}$, we have $P_{k} B(z)=0$, for $k=1, \cdots, r$. We proceed to show that $B(z)$ satisfies the condition of Lemma $1:\left(z^{n} B(z)\right)$ is an orthonormal set in $\mathscr{C}(z)$. It is to begin with clear that each element $z^{n} B(z)$ of the set has unit norm. To prove orthogonality, it must be shown that $\left\langle z^{m} B(z), z^{n} B(z)\right\rangle=$ 0 whenever $m=m_{1} \cdots m_{r}$ and $n=n_{1} \cdots n_{r}$ are unequal, i.e. whenever $m_{k} \neq n_{k}$ for at least one $k$. If $m_{i} \neq n_{i}$, all other indices being equal, we have

$$
\left\langle z^{m} B(z), z^{n} B(z)\right\rangle=\left\langle z_{i}^{m_{i}} B(z), z_{i}^{n_{i}} B(z)\right\rangle=0,
$$

since it is clear that each of the sets $\left(z_{k}^{n} B(z)\right)(k=1, \cdots, r)$ is orthonormal in $\mathscr{C}(z)$. If exactly two indices $i, j(j>i)$ differ, with say $m_{i}-n_{i}=\mu>0$, and $n_{j}-m_{j}=v>0$, we have

$$
\begin{aligned}
\left\langle z^{m} B(z), z^{n} B(z)\right\rangle & =\left\langle z_{i}^{\mu} B(z), z_{j}^{v} B(z)\right\rangle \\
& =\left\langle P_{i} z_{i}^{\mu} B(z), z_{j}^{v} B(z)\right\rangle=\left\langle z_{i}^{\mu} B(z), P_{i} z_{j}^{v} B(z)\right\rangle=0,
\end{aligned}
$$

since $P_{i} z_{j}^{v} B(z)=z_{j} P_{i} z_{j}^{v-1} B(z)=\cdots=z_{j}^{v} P_{i} B(z)$ and $P_{i} B(z)=0$. The extension to the general case is immediate.

Given the fact that $\left(z^{n} B(z)\right)$ is an orthonormal set, it next follows from Lemma 1 that $\mathscr{M}(B)$ is the closed span of elements $z^{n} B(z)$. Since $B(z)$ is in $\mathscr{M}$ and $\mathscr{M}$ is closed, $\mathscr{M}(B) \subseteq \mathscr{M}$. Before proceeding with the proof that $\mathscr{M}(B)=\mathscr{M}$, we may conveniently note that $\mathscr{B}$ is of dimension 1 . For if $A(z)$ and $B(z)$ are two elements of $\mathscr{B}$ having unit norm, the reasoning of the last paragraph shows that: (i) $\left(z^{n} A(z)\right)$ and $\left(z^{n} B(z)\right)$ are orthonormal sets in $\mathscr{C}(z)$; (ii) $\left\langle z^{m} A(z), z^{n} B(z)\right\rangle=0$ whenever $m \neq n$. It follows from Lemma 2 that $A(z)$ and $B(z)$ are linearly dependent in $\mathscr{C}(z): A(z)=c B(z)$, where $c$ is a complex constant of absolute value 1 .

To complete the proof, we show that $\mathscr{L}$, the orthogonal complement of $\mathscr{M}(B)$ in $\mathscr{M}$, has dimension 0 . Notice that $\mathscr{B} \subseteq \mathscr{M}(B), \mathscr{M} \cap \mathscr{B}^{\perp}=\mathscr{N}=$ $\bigvee_{k} \mathscr{N}_{k}$ and that

$$
\mathscr{L}=\mathscr{M}(B)^{\perp} \cap \mathscr{M} \subseteq \mathscr{B}^{\perp} \cap \mathscr{M}=\bigvee_{k} \mathscr{N}_{k} .
$$


We begin by proving that $\mathscr{L}$ is invariant under each $P_{j}$. For this it is enough to show that if $h(z)$ is in $\mathscr{M}(B)$, so is $P_{j} h(z)$. By linearity and continuity we may assume that $h(z)=z^{n} B(z)$ for some multi-index $n$. If $n_{j}>0$, then $P_{j} z^{n} B(z)=z^{n} B(z)$. If $n_{j}=0$, the commutativity hypothesis gives $P_{j} z^{n} B(z)=z^{n} P_{j} B(z)=0$.

Now let $f(z)$ belong to $\mathscr{L}$. Since $\mathscr{L} \subseteq \mathscr{N}$, the reasoning which led to equations (2), (3) above gives

$$
f(z)=P f(z)=P_{1} f(z)+P_{2} f(z)-P_{1} P_{2} f(z)
$$

and

$$
f(z)=z_{1} f_{1}(z)+z_{2} f_{2}(z)-z_{1} z_{2} f_{12}(z)
$$

where $f_{1}(z), f_{2}(z), f_{12}(z)$ are in $\mathscr{M}$. By the result of the last paragraph, $P_{1} f(z)=z_{1} f_{1}(z)$ belongs to $\mathscr{L}$. Thus for any $h(z)$ in $\mathscr{M}(B)$,

$$
\left\langle f_{1}(z), h(z)\right\rangle=\left\langle z_{1} f_{1}(z), z_{1} h(z)\right\rangle=\left\langle P_{1} f(z), z_{1} h(z)\right\rangle=0
$$

since $\mathscr{M}(B)$ is an ideal. Therefore $f_{1}(z)$ is in $\mathscr{L}$. Similarly $f_{2}(z)$ and $f_{12}(z)$ belong to $\mathscr{L}$. The representation (3.1) holds, where $f_{1}(z), f_{2}(z), f_{12}(z)$ belong to $\mathscr{L}$. Since $f_{1}(z), f_{2}(z)$ and $f_{12}(z)$ have representations of the type (3.1), we can repeat the argument. After $n$ repetitions we have (cf. (4))

$$
f(z)=\sum q_{\alpha}(z) f_{\alpha}(z)
$$

where $q_{\alpha}(z)$ is a monomial of degree $\geqq n$ and $f_{\alpha}(z) \in \mathscr{L}$ for all $\alpha$. Therefore $f(z)$ vanishes identically, and $\mathscr{L}=(0)$ as claimed.

ACKNOWLEDGEMENT. I am deeply grateful to the referee for suggestions which I have adopted in the proofs that $\mathscr{M} \neq \mathscr{N}$, and that $\mathscr{L}=(0)$.

\section{REFERENCES}

1. A. Beurling, On two problems concerning linear transformations in Hilbert space, Acta Math. 81 (1948), 17 pp. MR 10, 381.

2. L. deBranges and J. Rovnyak, Square summable power series, Holt, Rinehart and Winston, New York, 1966. MR 35 \#5909.

3. J. von Neumann, Functional operators. II. The geometry of orthognal spaces, Ann. of Math. Studies, no. 22, Princeton Univ. Press, Princeton, N.J., 1950. MR 11, 599.

4. J. Rovnyak, Ideals of square summable power series, Math. Mag. 33 (1959/60), 265-270. MR 23 \#A1227.

Department of Mathematics, University of New Hampshire, Durham, New HAMPSHIRE 03824 